\title{
Successful Treatment of a Cervical Molar Pregnancy: Combined Medical and Surgical Methods
}

\author{
Azam Manshadi Tarafdari ${ }^{1}$, Fatemeh Farajzadeh Vajari ${ }^{2 *}$ and Shahrzad Sheikhhasani ${ }^{1 *}$ \\ ${ }^{1}$ Assistant Professor of obstetrics and gynecology, fellowship in IVF and infertility.Imam Khomeini Hospital Complex (IKHC). Tehran \\ University of Medical Sciences, Tehran, Iran
}

${ }^{2}$ Fellowship in IVF \& infertility, obstetrics \& gynecology department, Iran

*Corresponding author: Fatemeh Farajzadeh Vajari, Fellowship in IVF \& infertility, obstetrics \& gynecology, Imam Khomeini Hospital Complex, Tehran University of Medical Sciences, Iran

Shahrzad Sheikhhasani, Assistant professor of obstetrics and gynecology department, Fellowship in gynecological oncology, Imam Khomeini Hospital Complex, Tehran University of Medical Sciences, Tehran, Iran

\begin{abstract}
ARTICLE INFO
Received: 幽 December 13, 2019

Published: 絊January 06, 2020

Citation: Azam Manshadi Tarafdari, Fatemeh Farajzadeh Vajari, Shahrzad Sheikhhasani. Successful Treatment of a Cervical Molar Pregnancy: Combined Medical and Surgical Methods. Biomed J Sci \& Tech Res 24(1)-2020. BJSTR. MS.ID.004009.
\end{abstract}

Keywords: Ectopic Pregnancy; Hydatidiform Mole; Methotrexate

\section{ABSTRACT}

Background: Cervical Ectopic Pregnancy (CEP) is a rare kind of ectopic pregnancy particularly if being Hydatidiform mole. Molar ectopic pregnancy treatment is challenging especially with intention to preserve the fertility. Here we report our challenge to preserve fertility and treatment of a partial molar cervical pregnancy.

Case Presentation: A 37-year-old primigravid lady presented with vaginal bleeding with stable vital signs. $\beta$ HCG level was disproportionately high (i.e. 113000 $\mathrm{mIU} / \mathrm{mL}$ at 7 weeks of gestation according to CRL). Ultrasonography was suggestive of partial hydatidiform mole due to thickened hyper vascular multi cyctic placenta with hydropic changes and with fetal heart activity. We considered treatment as less- invasive approach with fetal reduction (i.e. KCL injection) and chemotherapy followed by suction and curettage with additional cervical Foley catheter inflation. Uterine was preserved with no need for transfusion. Patient was followed by weekly $\beta$ HCG measurements to undetectable levels and then monthly for 6 months. Pathology was confirmatory for the diagnosis of hydatidiform mole.

Principal Conclusion: Managing cervical ectopic pregnancy in a primigravid patient is challenging to simultaneously save patient's life and childbearing ability. Combined chemotherapy and surgical treatments could be considered promising.

\section{Introduction}

Ectopic pregnancy is a critical diagnosis in any woman presenting with vaginal bleeding and lower abdominal pain. Cervical Ectopic Pregnancy (CEP) is a rare kind of ectopic pregnancy in which implantation occurs in the endocervical canal rather than in the uterus and includes less than $1 \%$ of all ectopic pregnancies $[1,2]$. It happens mostly in smokers, IVF pregnancies, and in those with exposure to DES, history of previous cesarean surgery, and history of pelvic inflammatory disease [2]. Under-diagnosis can be life threatening and should be concisely considered. Hydatidiform Mole (HM) is a premalignant type of gestational trophoblastic disease that happens from inappropriate fetal and placental development. Partial HM develops after fertilization of ovum by either two sperm or one diploid sperm causing a triploid mole (69XXX, 69XXY, or 69XYY). In such instances, fetal cardiac activity can be detected. Partial or complete mole affects approximately one in 500-1000 pregnancies [3]. Though, ectopic pregnancy and molar pregnancy are not rare and occur approximately twenty in every 1000 and one in every 500 to 1000 pregnancies, respectively, the combination of them (i.e. an ectopic HM) is uncommon. Reports regarding molar ectopic pregnancy are sparse [4]. Here, we want to present a rare case of cervical pregnancy, which was molar pregnancy and demonstrate our therapeutic strategy to preserve her fertility. 


\section{Case Presentation}

On 21 April 2019, a 37-year-old primigravid lady admitted to the Imam Khomeini Hospital Complex, Tehran University of Medical Sciences, Iran. She was complaining of vaginal bleeding which was continued after her last menstrual period. On physical examination, she had stable vital signs (pulse rate: 90/min, BP: 110/70 mmHg, Respiratory rate: $21 / \mathrm{min}$, and oral temperature: $37.1^{\circ} \mathrm{C}$ ). Systemic examination illustrated no unusual findings. During gynecologic examination, external os was open with scant bleeding. $\beta$ HCG was positive and its titration upon admission was $113000 \mathrm{mIU} /$
$\mathrm{mL}$; Hemoglobin was $10.8 \mathrm{~g} / \mathrm{dL}$ and liver and renal function tests and thyroid-stimulating hormone were within normal limits. Ultrasonography revealed an enlarged uterus and distended cervix with gestational sac 7 weeks based on CRL and fetus was detected with fetal heart activity. Pregnancy product was surrounded by Placenta circuit (Figure 1). Ultrasonography examination detected a fetus with fetal heart activity (hence complete mole is excluded) in addition to thickened multi cystic placenta with hydropic changes and hyper vascularization in Doppler mode which in addition of high $\beta$ HCG level (113000 mIU/mL) was suggestive of partial hydatidiform mole.

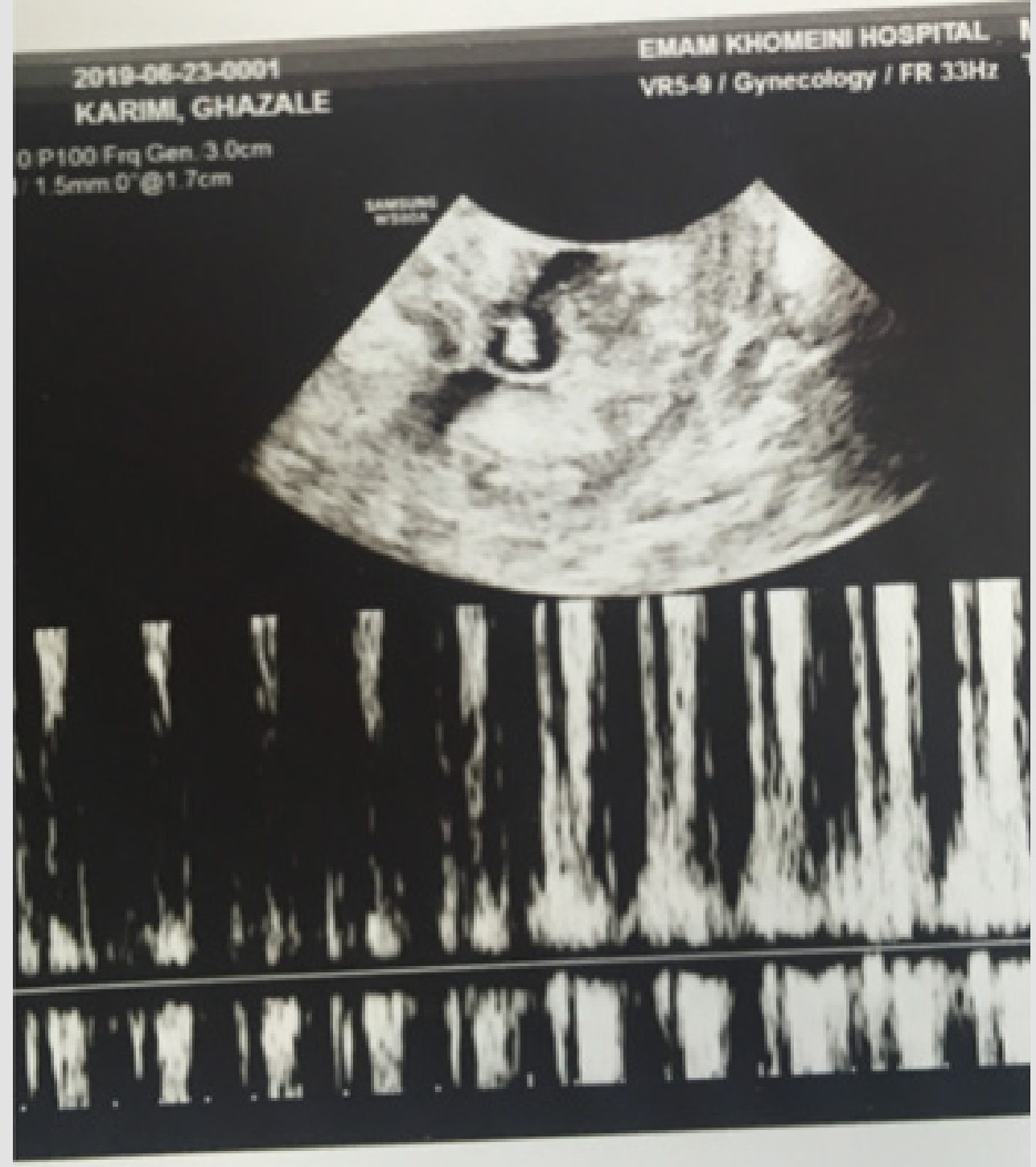

Figure 1: Pregnancy product was surrounded by Placenta circuit. 
Patient was admitted with suspicious cervical molar pregnancy. We planned to save uterus employing fetal reduction and chemotherapy in this primigravid lady. Consequently firstly, we tried to do fetal reduction with injection of 3cc kcl 15\% under ultrasonographic guide. Chemotherapy was scheduled for multi dose injection of methotrexate: $1 \mathrm{mg} / \mathrm{kg}$ on Days 1, 3, 5, 7 in addition to folinic acid $0.1 \mathrm{mg} / \mathrm{kg}$ on Days 2, 4, 6,8. Although, after the fourth dose of methotrexate, $\beta$ HCG titer increased to 154800 but in color Doppler ultrasonography, the placental vascularity had been decreased. Then we contented patient about probable complications and proceeded to execute suction curettage. All the pregnancy products were removed and to prevent excessive bleeding a Foley catheter was inflated in the cervix. Patient did not need blood transfusion. Eventually, $\beta$ HCG level dropped under 15000 upon operation. Patient was discharged with advice for barrier contraception, considering she was hemodynamically stable and had trace vaginal bleeding. Pathology examination indicated presence of a typical trophoblastic proliferation, decidualized tissue and degenerative villi. The findings were reported compatible with hydatidiform mole, recommending serial bHCG serologic tests. Serial serum B-hCG gradually decreased through weekly $\beta$ HCG measurement to undetectable levels within a span of 6 weeks and we followed her for 6 months later.

\section{Discussion}

There is no consensus on the treatment strategy for cervical pregnancy and particularly cervical molar pregnancy. In the current report, we provide our successful experience in the treatment of the condition intending to preserve the fertility. Any cervical pregnancy should be terminated; surgical interventions could be delayed in stable patients. Our method including fetal reduction and chemotherapy could have been curative, but not sufficient in the current case which leaded us to perform suction and curettage. Prior chemotherapy reduced the vascularity assessed by Doppler method and possibly assisted the intension to preserve uterine. Furthermore, the report of this case is important reminding the possibility of molar pregnancies in ectopic pregnancies. Molar pregnancies can be neglected or misdiagnosed as missed abortions or clotted blood, which highlights the significance of high clinical suspicion [5]. Despite unknown exact etiologies of a molar pregnancy, remains unknown, certain epidemiologic features of complete and partial mole differ markedly [6].

Use of oral contraceptives and a history of irregular menstruation can be assumed risk factors of partial mole; however, dietary factors have no role in this regard. Although EP and molar pregnancies rarely occur, almost 40 cases of hydatidiform mole have been reported in the previous studies [7]. And according to our extended search in published articles, we found rare tubal molar pregnancies, though our presentation shows a cervical molar pregnancy. Abdominal pain and vaginal bleeding are the most common symptoms of HM [8]. The features that are usual symptoms of an ectopic pregnancy, as in the current case. Consequently, any similar history should evoke consideration. Sonographey could be a method of choice. "Snow-storm", "cluster of grapes" or "honeycomb" are classic features of complete mole, whereas in partial mole, more focal lesions are detected. Complete and partial HM could be discriminated mainly based on the presence of fetal tissue in partial HM [5]. Signs, symptoms or laboratory tests could not discriminate molar EP from cervical pregnancy. In the current report, patient had a high $\beta$ HCG levels at 7 weeks of gestation and gestational sac with fetal heart activity in endo-cervix surrounded by hyper vascular multicystic placenta with hydropic changes in ultrasound examination.

These findings remarkably increased the possibility of partial HM. Cervical pregnancy should be terminated as was performed for the patient. We believe that the findings of pathology, although could be subject to uncertainty due to prior chemotherapy, confirms HM was the underlying cause in this patient. Nevertheless, ploidy study, we did not perform, is the ultimate and only certain method to confirm the etiology of the pathology. In a multiparous patient, Houssine Boufetta et al. reported a complete mole in a cervical pregnancy which was treated with hysterectomy and chemotherapy [9]. In a similar report by Keitaroh Takeda et al. [2] cervical ectopic molar pregnancy in a multiparous patient was managed with Uterine artery embolization and chemotherapy [2]. Our patient had no child and saving childbearing ability was prioritized for us and we had to change our approach to save uterus if possible. We considered a non- invasive approach with fetal reduction and chemotherapy which followed by suction- curettage.

\section{Conclusion}

Ectopic partial molar pregnancy as a rare diagnosis, needs high clinical suspicion for diagnosis. High serum $\beta$ HCG levels and confirmatory findings in ultrasonography increase the suspicion level. Surely, pathological examination is essential to reach final diagnosis. Preservation of fertility is possible when required employing fetal reduction and chemotherapy.

\section{Acknowledgment}

This research was not funded by the government or a private sector.

\section{Conflicts of Interest}

There are no conflicts of interest.

\section{References}

1. Dhiilon R, Pai H, Gandhi NS, Singh N (2019) Ectopic pregnancy, Principle and practice. Assisted Reproductive technology pp. 864-865.

2. Takeda K, Mackay J, Watts S (2018) Successful Management of Cervical Ectopic Pregnancy with Bilateral Uterine Artery Embolization and Methotrexate. Case Rep Emerg Med p. 4.

3. Stevens FT, Katzorke N, Tempfer C, Kreimer U, Bizjak GI, et al. (2015) Gestational trophoblastic disorders: an update in 2015. Geburtshilfe and Frauenheilkunde 75(10): 1043-1050. 
4. Allen L, Dawson C, Nascu P, Rouse T (2016) A Molar Pregnancy within the Fallopian Tube. Case Rep Obstet Gynecol 4367181

5. Marion Vincent Mumping, Poonam Desai, Nadia Maria Shaukat (2017) Partial Hydatidiform Molar Pregnancy.

6. Worley MJ, Joseph NT, Berkowitz RS, Goldstein DP (2014) Women with a partial mole during their first pregnancy and diagnosed earlier in gestation are at increased risk of developing gestational trophoblastic neoplasia. International Journal of Gynecological Cancer 24(5): 941-945

7. Beena D, Tirthanath S, Jose V, Shetty J (2016) Molar pregnancy presents as tubal ectopic pregnancy: a

\section{ISSN: 2574-1241}

DOI: $10.26717 /$ BJSTR.2020.24.004009

Fatemeh Farajzadeh V, Shahrzad S. Biomed J Sci \& Tech Res

(C) (P) This work is licensed under Creative

Submission Link: https://biomedres.us/submit-manuscript.php rare case report. Journal of Clinical and Diagnostic Research: JCDR 10(1): 10.

8. American M, Hasanzadeh M, Hafizi L (2017) Ectopic Partial Molar Pregnancy: A Case Report. Journal Midwifery Reproductive of Health 5(1): 848-850.

9. Boufettal H, Samouh N (2018) Invasive hydatidiform mole in the cervix. Pan African Medical Journal 29-27.

$\begin{array}{ll}\text { BIOMEDICAL } & \text { Assets of Publishing with us } \\ \text { RESEARCHES } & \text { - Global archiving of articles } \\ \text { - Immediate, unrestricted online access } & \text { - Rigorous Peer Review Process } \\ & \text { - Authors Retain Copyrights }\end{array}$

